\title{
Structure analysis of the extracellular domain reveals disulfide bond forming-protein properties of Mycobacterium tuberculosis Rv2969c
}

\author{
Lu Wang ${ }^{1,2}$, Jun Li ${ }^{1,2}$, Xiangxi Wang ${ }^{1,2}$, Wu Liu ${ }^{1}$, Xuejun C. Zhang ${ }^{1}$, Xuemei Li ${ }^{1}$, Zihe Rao ${ }^{1,3} \bowtie$ \\ ${ }^{1}$ National Laboratory of Biomacromolecules, Institute of Biophysics, Chinese Academy of Sciences, Beijing 100101, China \\ ${ }^{2}$ University of Chinese Academy of Sciences, Beijing, China \\ ${ }^{3}$ Tianjin Key Laboratory of Protein Science, College of Life Sciences, Nankai University, Tianjin 300071, China \\ $\triangle$ Correspondence: raozh@xtal.tsinghua.edu.cn \\ Received April 21, 2013 Accepted May 28, 2013
}

\begin{abstract}
Disulfide bond-forming (Dsb) protein is a bacterial periplasmic protein that is essential for the correct folding and disulfide bond formation of secreted or cell wallassociated proteins. DsbA introduces disulfide bonds into folding proteins, and is re-oxidized through interaction with its redox partner DsbB. Mycobacterium tuberculosis, a Gram-positive bacterium, expresses a DsbA-like protein (Rv2969c), an extracellular protein that has its $\mathrm{N}$ terminus anchored in the cell membrane. Since Rv2969c is an essential gene, crucial for disulfide bond formation, research of DsbA may provide a target of a new class of anti-bacterial drugs for treatment of M.tuberculosis infection. In the present work, the crystal structures of the extracellular region of Rv2969c (Mtb DsbA) were determined in both its reduced and oxidized states. The overall structure of Mtb DsbA can be divided into two domains: a classical thioredoxin-like domain with a typical CXXC active site, and an $\alpha$-helical domain. It largely resembles its Escherichia coli homologue EcDsbA, however, it possesses a truncated binding groove; in addition, its active site is surrounded by an acidic, rather than hydrophobic surface. In our oxidoreductase activity assay, Mtb DsbA exhibited a different substrate specificity when compared to EcDsbA. Moreover, structural analysis revealed a second disulfide bond in Mtb DsbA, which is rare in the previously reported DsbA structures, and is assumed to contribute to the overall stability of Mtb DsbA. To investigate the disulphide formation pathway in M.tuberculosis, we
\end{abstract}

modeled $M$ tb Vitamin K epoxide reductase (Mtb VKOR), a binding partner of Mtb DsbA, to Mtb DsbA.

KEYWORDS Mycobacterium tuberculosis, disulfide bond forming protein, X-ray crystallography

\section{INTRODUCTION}

Mycobacterium tuberculosis (Mtb) is a Gram-positive bacterium, and the causative agent of Tuberculosis (TB), being the most wide-spread infectious disease on the planet, affecting approximately $30 \%$ of the world's population (Yang et al., 2011). In the year of 2011, about 8.7 million people were infected with TB, resulting in 1.4 million deaths (WHO 2012, Global Tuberculosis Control Report, http://www.who.int/tb/publications/ global_report/en/). Currently, the resistance to clinically used drugs, and the co-infection with HIV/HCV present a major challenge to TB therapy (Comas and Gagneux, 2009). The virulence and pathogenicity of Mtb is closely related to its secreted proteins, which are either cell wall-associated or extracellular. About 60 percent of the secreted proteins have disulfide bonds (Goulding et al., 2003), the formation of which is crucial for protein folding and function. Thus, the research of the structure and function of disulfide bond forming (Dsb) proteins may provide a way to identify new anti-TB drug targets, and overcome increasingly serious multidrug-resistant TB (Goulding et al., 2002).

Dsb proteins are well-studied in Escherichia coli (E.coli), which is a Gram-negative bacterium (Messens and Collet, 2006). In its periplasmic space, oxidative E.coli DsbA (EcDsbA) 
transfers its disulfide bonds to its substrates, and is re-oxidized by its partner membrane protein E.coli DsbB (EcDsbB), which in turn is then re-oxidized by ubiquinone present in the cytoplasm (Inaba and Ito, 2008). E.coli DsbC (EcDsbC) (McCarthy et al., 2000) and E.coli DsbG (EcDsbG) (Shao et al., 2000) can form homodimers, and both possess disulfide bond isomerase activity. E.coli DsbE (EcDsbE) (also known as CcmG, or Cytochrome c biogenesis protein) (Reid et al., 2001) is involved in the maturation of Cytochrome c. E.coli DsbD (EcDsbD) is a transmembrane protein, whose function is to transfer reducing equivalents from the cytoplasmic thioredoxin system to the periplasm, to maintain EcDsbC, EcDsbE and EcDsbG in their reduced states (Stewart et al., 1999; Denoncin and Collet, 2012).

Gram-positive bacteria do not have the periplasmic space of Gram-negative bacteria, which requires Gram-positive bacteria to utilize a different mechanism for protein disulfide bond formation (Daniels et al., 2010). For example, the genome of Bacillus subtilis encodes a set of proteins corresponding to the EcDsb proteins. BdbD (bacillus disulfide bond protein), a membrane-anchored DsbA-like protein, introduces disulfide bonds to the proteins on the outside of the cytoplasmic membrane (Meima et al., 2002). BdbD is re-oxidized by BdbC, which has a sequence identity of $40 \%$ with DsbB, and which plays a similar role (Dorenbos et al., 2002). Another two membraneanchored proteins, ResA (Erlendsson et al., 2003) and StoA (Crow et al., 2009b), which are involved in Cytochrome c maturation and endospore biogenesis respectively, are reduced by $\mathrm{CcdA}$, a membrane protein that functions as EcDsbD (Erlendsson and Hederstedt, 2002). Some other bacteria, including $M$. tuberculosis and Synechococcus sp., contain DsbA homologs but lack a homologue of DsbB (Dumoulin et al., 2005). Meanwhile, their genomes encode a membrane protein VKOR, which is a homologue of vertebrate Vitamin $\mathrm{K}$ epoxide reductase, to functionally replace EcDsbB (Dutton et al., 2008). The overall structure of Synechococcus sp. VKOR (SpVKOR) (Li et al., 2010), which contains both a VKOR and a Trx-like domain, shows some distinct features compared to the structure of EcDsbB.

To date, the Dsb proteins identified in M.tuberculosis are Mtb DsbE, a DsbA-like protein (Goulding et al., 2004), its homologue named Mtb DsbF (Chim et al., 2010), its potential redox membrane protein partner Mtb DsbD (Goldstone et al., 2005) and Mtb VKOR (Wang et al., 2011). In the genome of $M$. tuberculosis, the gene directly adjacent to VKOR encodes an apparent DsbA-like protein (Rv2969c), which was predicted to have an $\mathrm{N}$-terminal transmembrane helix. Because of the difficulties in obtaining crystals of single transmembrane protein, together with the fact that sequence analysis suggested that the extracellular part of Rv2969c is the functional domain, we chose residues 53-255 (Mtb DsbA) for our structural and functional studies.

In the present work, we solved the high-resolution crystal structure of the functional domain of Rv2969c, in both its reduced and oxidized states. The overall structure shares similarity with that of $\mathrm{BdbD}$; however, it contains a second disulfide bond, which is likely to play a structural role, and which lacks a $\mathrm{Ca}^{2+}$-binding site. Moreover, there are several differences between the structures of Mtb DsbA and EcDsbA, with the latter thoroughly characterized by now. In vitro oxidoreductase activity assays, which use RNase and insulin as substrates, show different substrate specificities for Mtb DsbA and EcDsbA. Based on the structure of SpVKOR, and the local sequence identity of $30 \%$ (28/94) between Mtb VKOR and SpVKOR, we decided to use computational structural biology to construct the model of Mtb DsbA-VKOR complex, which will help explain their interaction mode, and reveal the disulphide formation pathway in M.tuberculosis. According to this model and previous analysis of $M t b$ VKOR, we propose a disulphide formation pathway for M.tuberculosis.

\section{RESULTS}

\section{Full-length Rv2969c is a single transmembrane protein}

Rv2969c contains 255 amino acid residues with an N-terminal transmembrane sequence. Sequence analysis suggested that one transmembrane helix locates at residues $20-42$, and that the extracellular part consisted of residues 43-255. When we expressed the full-length gene of Rv2969c in E.coli, the target protein was detected in the membrane fraction and only soluble in solution with detergent, which is consistent with the location of the transmembrane helix at the $\mathrm{N}$-terminus. Due to the low yield of full-length protein and the difficulties in crystallization, we crystallized residues 53-255, to investigate how Rv2969c catalyzes disulfide bond formation.

\section{The overall structure of Mtb DsbA}

The crystal structure of the reduced $M t b$ DsbA was determined using the Selenomethionine single-wavelength Anomalous Diffraction (SAD) method, and was refined to $1.90 \AA$ resolution, with R-work and R-free values of $16.6 \%$ and $20.6 \%$, respectively. The structure of the oxidized form was solved by molecular replacement, which used the reduced structure as a model, and was refined to $2.50 \AA$ resolution, with R-work and $\mathrm{R}$-free values of $19.8 \%$ and $22.3 \%$, respectively. The reduced Mtb DsbA crystal belongs to space group $P 2_{1} 2_{1} 2_{1}$, with unit cell parameters $a=46.9 \AA, b=74.9 \AA, c=119.9 \AA$ (Table 1). In one asymmetric unit, there is a $\mathrm{SO}_{4}{ }^{2-}$ molecule, which forms salt bridges with Lys ${ }^{159}$ and Arg $^{97}$ in chain A and Lys ${ }^{235^{\prime}}$ in the other protomer and contributes to the crystallization packing. Due to the high resolution of the crystal structure, we were able to observe alternative conformations of the side chains of several amino acid residues in the structure.

According to Matthews coefficient analysis (Matthews, 1968), there are two molecules in one asymmetric unit, at a solvent content of $51 \%\left(\mathrm{VM}=2.5 \AA^{3} \mathrm{Da}^{-1}\right)$. PISA analysis (Krissinel and Henrick, 2007) indicated that the largest interface in the lattice buries only $480 \AA^{2}$ of surface area. Also, the complexion significance score is 0.0 , which indicates that the protein cannot form stable dimers in solution. The result of an- 
Table 1. Summary of data processing and refinement statistics

\begin{tabular}{|c|c|c|}
\hline Name & Mtb DsbA_Se_reduced & Mtb DsbA oxidized \\
\hline PDB entry & 4JR6 & 4JR4 \\
\hline \multicolumn{3}{|l|}{ Data collection } \\
\hline Wavelength $(\AA)$ & 0.9790 & 0.9800 \\
\hline Resolution (Å) & $50.00-1.90(1.97-1.90)$ & $50.00-2.50(2.59-2.50)$ \\
\hline Space group & $P 2{ }_{1} 2_{1} 2_{1}$ & $P 2_{1} 2_{1} 2_{1}$ \\
\hline Unit cell parameters $\left(\AA,{ }^{\circ}\right)$ & $\begin{array}{l}a=46.9, b=74.9, c=119.9 \\
\alpha=\beta=\gamma=90.0\end{array}$ & $\begin{array}{l}a=46.7, b=75.0, c=119.7 \\
\alpha=\beta=\gamma=90.0\end{array}$ \\
\hline \multicolumn{3}{|l|}{ No. of reflections } \\
\hline Total observation & 233,030 & 97,878 \\
\hline Unique reflections & $63,755(6225)$ & $14,886(1,456)$ \\
\hline Redundancy & $6.9(7.2)$ & $6.6(6.6)$ \\
\hline Completeness (\%) & $99.9(100.0)$ & $97.7(97.8)$ \\
\hline$R_{\text {merge }}{ }^{\mathrm{a}}$ & $0.128(0.439)$ & $0.141(0.519)$ \\
\hline$\| / \sigma(I)$ & $13.5(5.0)$ & $8.6(3.9)$ \\
\hline \multicolumn{3}{|l|}{ Refinement } \\
\hline No. of reflections used & 61,490 & 14,267 \\
\hline$R_{\text {work }}^{\mathrm{b}}$ & 0.166 & 0.198 \\
\hline$R_{\text {free }}{ }^{\mathrm{c}}$ & 0.206 & 0.223 \\
\hline \multicolumn{3}{|l|}{ Geometry deviations } \\
\hline Bond lengths $(\AA)$ & 0.019 & 0.004 \\
\hline Bond angles $\left({ }^{\circ}\right)$ & 1.352 & 0.826 \\
\hline \multicolumn{3}{|l|}{ No. of non-H atoms } \\
\hline Protein & 3,003 & 2,930 \\
\hline Ligand & 5 & 20 \\
\hline Water & 452 & 90 \\
\hline Average $B$ factor $\left(\AA^{2}\right)$ & 25.72 & 29.44 \\
\hline Protein & 24.33 & 28.20 \\
\hline Ligand & 23.33 & 48.71 \\
\hline Solvent & 34.96 & 26.78 \\
\hline \multicolumn{3}{|l|}{ Ramachandran plot (\%) } \\
\hline Most favored & 99.0 & 98.2 \\
\hline Additionally allowed & 1.0 & 1.8 \\
\hline Outlier & 0.0 & 0.0 \\
\hline
\end{tabular}

Values in parentheses are for highest-resolution shell.

${ }^{\mathrm{a}} R_{\text {merge }}=\Sigma \mathrm{hkl} \Sigma \mathrm{i}|\mathrm{l}(\mathrm{hkl}) \mathrm{i}-<|(\mathrm{hkl})>\mid / \Sigma \mathrm{hkl} \Sigma \mathrm{i} \mathrm{l}(\mathrm{hkl}) \mathrm{i}$.

${ }^{\mathrm{b}} R_{\text {work }}=\Sigma \mathrm{hkl}|\mathrm{Fo}(\mathrm{hkl})-\mathrm{Fc}(\mathrm{hkl})| / \Sigma \mathrm{hkl}$ Fo (hkl).

${ }^{\mathrm{c}} R_{\text {free }}$ was calculated for a test set of reflections (5\%) omitted from the refinement.

alytical ultracentrifugation indicated that in solution, Mtb DsbA predominantly exists in its monomeric form. The dimers we observed in crystals may have resulted from the crystallographic packing, likely to be of no biological significance. Furthermore, their structures are nearly identical, with a root mean square deviation (r.m.s.d.) of $0.6 \AA$ between $196 \mathrm{C} \alpha$ atom pairs. Thus, our discussion below is predominantly based on the structure of one arbitrarily chosen protein protomer, unless stated otherwise. The overall structure of Mtb DsbA can be divided into two domains: a classical thioredoxin-like domain (Trx domain) and an $\alpha$-helical domain (helical domain) (Fig. 1). The Trx domain has a typical thioredoxin fold, and is composed of a $\beta \alpha \beta$ motif

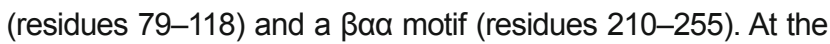
$\mathrm{N}$-terminus of the first helix of the Trx domain is a CXXC motif $\left(\right.$ Cys $^{89}{ }^{8}$ Pro $^{90}$-Ala ${ }^{91}$-Cys ${ }^{92}$ ), suggesting that Rv2969c is a redoxactive Trx-like protein. The helical domain consists of five $\alpha$-helices, three of which ( $\alpha 2-\alpha 4)$ form an antiparallel helical bundle and the other $\alpha$-helix ( $a 6)$ connects the $\beta a \alpha$ motif. 


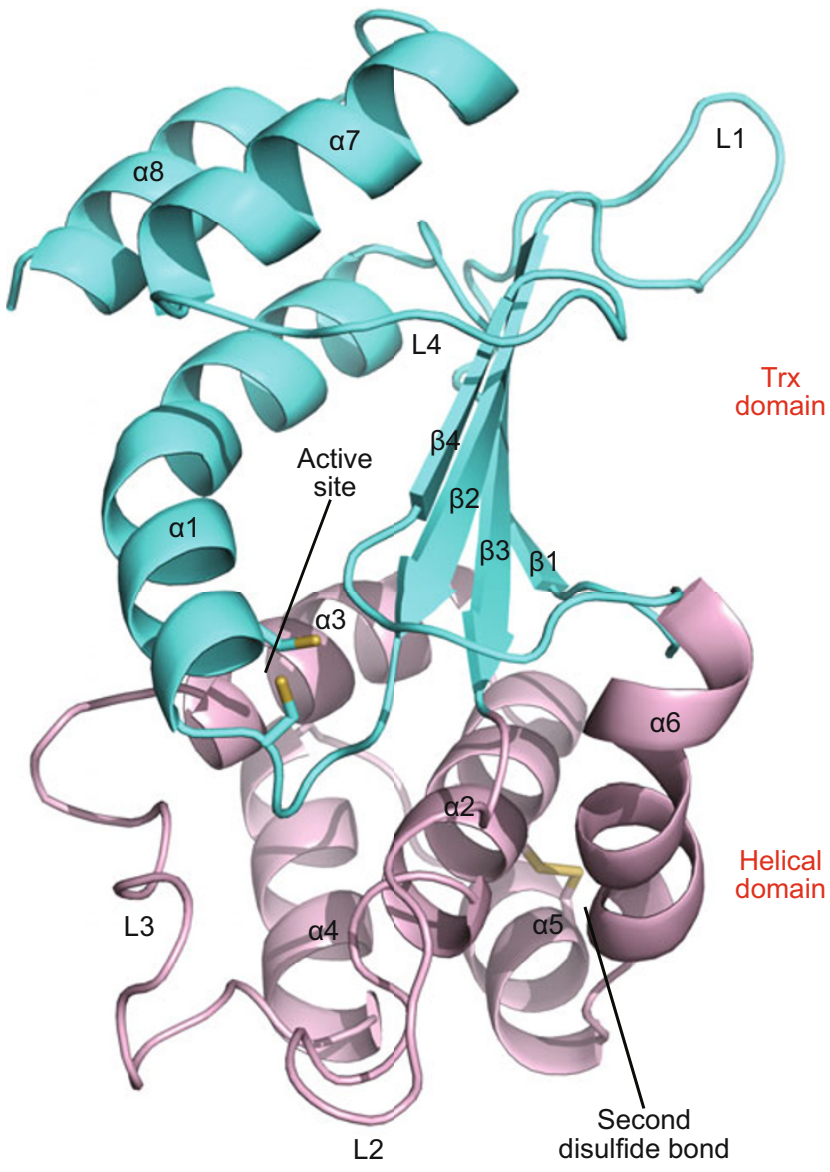

Figure 1. Structure of the extracellular region of Mtb DsbA. The overall structure of Mtb DsbA can be divided into two domains: a classical thioredoxin-like domain (cyan) and an $\alpha$-helical domain (pink).

\section{Comparison with homologous DsbA structures}

The result of a Dali search indicated that the crystal structure of Mtb DsbA is most similar to Bacillus subtilis BdbD (PDB: 3EU3) (Crow et al., 2009a); however, it lacks the metal ion binding site that was expected to stabilize the reduced form of the active site. The r.m.s.d. between them was $2.3 \AA$ for 161 $\mathrm{C} \alpha$ of aligned residues, and the sequence identity is $28 \%$ (Sequence alignment see Fig. 2). The overall folds of Mtb DsbA resemble that of EcDsbA (PDB: 1DSB) (Martin et al., 1993); the $\mathrm{C} \alpha$ atoms of 130 residues of Mtb DsbA can be superimposed with an r.m.s.d. of $2.9 \AA$. Despite the overall similarities, there are several notable structural differences between $M t b$ DsbA and EcDsbA, including the regions surrounding the active site, which may affect substrate specificity and activity, or could be modulating the redox characteristics.

Firstly, Mtb DsbA contains four $\beta$-strands, three of which make up the central $\beta$-sheet (i.e. strands $\beta 2, \beta 3$ and $\beta 4$ ). In contrast, in other DsbA family proteins, the central $\beta$-sheet is generally composed of four $\beta$-strands. Besides, the orientation and location of $\mathrm{N}$ terminal strand $\beta 1$ is topologically different from the corresponding region in EcDsbA (Fig. 3A). In Mtb DsbA, strand $\beta 1$ is anti-parallel to strand $\beta 3$, which approaches the helical domain, while in EcDsbA, strand $\beta 1$ is anti-parallel to strand $\beta 5$, which approaches the $C$ terminal helix. Therefore, the position of the loop1, which connects $\beta 1$ and $\beta 2$, is considerably different from the equivalent loop1 observed in EcDsbA. Loop1 is approaching the $\alpha 7$ of the Trx domain in Mtb DsbA, while loop1 is approaching the $a 6$ helix of the helical domain in EcDsbA. Secondly, loop2 connecting $\beta 3$ and $\alpha 2$ has four more residues than the equivalent loop2 observed in $\mathrm{EcDsbA}$. Compared with EcDsbA, loop3 linking $\alpha 3$ and $\alpha 4$ has three extra residues in Mtb DsbA and turns towards the CXXC active site, positioning the acidic side chain of $\mathrm{Glu}^{165}$ near the nucleophilic Cys $^{89}$ (6.26 A) (Fig. 3B). According to the sequence alignment, acidic residues are primarily in this loop3 in Gram-positive DsbAs, but are absent in EcDsbA (Fig. 2). Loop3 forms one edge of an acidic groove in Mtb DsbA. The proximity of $\mathrm{Glu}^{165}$ to the redox active site of $M t b$ DsbA suggests a possible role in substrate specificity, or in modulating the redox characteristics. Thirdly, the motif of $\beta 4 \alpha 7 \alpha 8$ and loop4 (between $\beta 4$ and $\alpha 7$ ) at the C-terminal end of the polypeptide chain produces a considerably difference between Mtb DsbA and EcDsbA (Fig. 3C). This region forms one edge of a hydrophobic peptide-binding groove in EcDsbA (Paxman et al., 2009) that binds to DsbB (Inaba et al., 2006) and is supposed to interact with unfolded protein substrates. In Mtb DsbA, this region is not hydrophobic, and is 9 residues shorter, resulting in a shorter loop connecting $\beta 4$ and $\alpha 7$, a shorter $\alpha 7$, and an extra $\alpha 8$ helix. Sequence alignment shows that this deletion appears to be conserved in DsbAs from Gram-positive organisms (Fig. 2). Finally, there is a second disulfide bond between helices $\alpha 2$ and a5 in Mtb $\mathrm{DsbA}$, which is highly conserved in a-proteobacterial DsbAs, but which is absent in EcDsbA and other DsbAs (Kurz et al., 2009). Thermal stability test (thermally-induced protein melting) (Ericsson et al., 2006) shows that the double mutant form of Mtb DsbA $(\mathrm{C} 140,192 \mathrm{~K})$ is less stable. Thus, we predicted that this disulfide bond is associated with the stability and integrity of the overall protein structure.

\section{Surface characteristics}

There are a hydrophobic peptide-binding groove and an acidic groove surrounding the $\mathrm{CXXC}$ motif on the surface of $M t b$ DsbA (Fig. 3D). By contrast, there are a hydrophobic patch and a peptide-binding groove composing a hydrophobic pocket in EcDsbA, which is thought to be vital for catalytic activity (Inaba et al., 2006). Comparing with the peptide-binding groove in EcDsbA, the peptide-binding groove in Mtb DsbA is shallower as well as smaller. Moreover, the hydrophobic patch adjacent to the active site in EcDsbA is absent in Mtb DsbA, but contains an acidic groove instead. Several residues that contribute to this patch in EcDsbA (Phe ${ }^{63}-\mathrm{Met}^{64}-\mathrm{Gly}^{65}-\mathrm{Gly}^{66}$ in the loop connecting the $\operatorname{Tr} x$ and helical domains) are replaced or covered with charged residues in Mtb DsbA. Thus, the differ- 

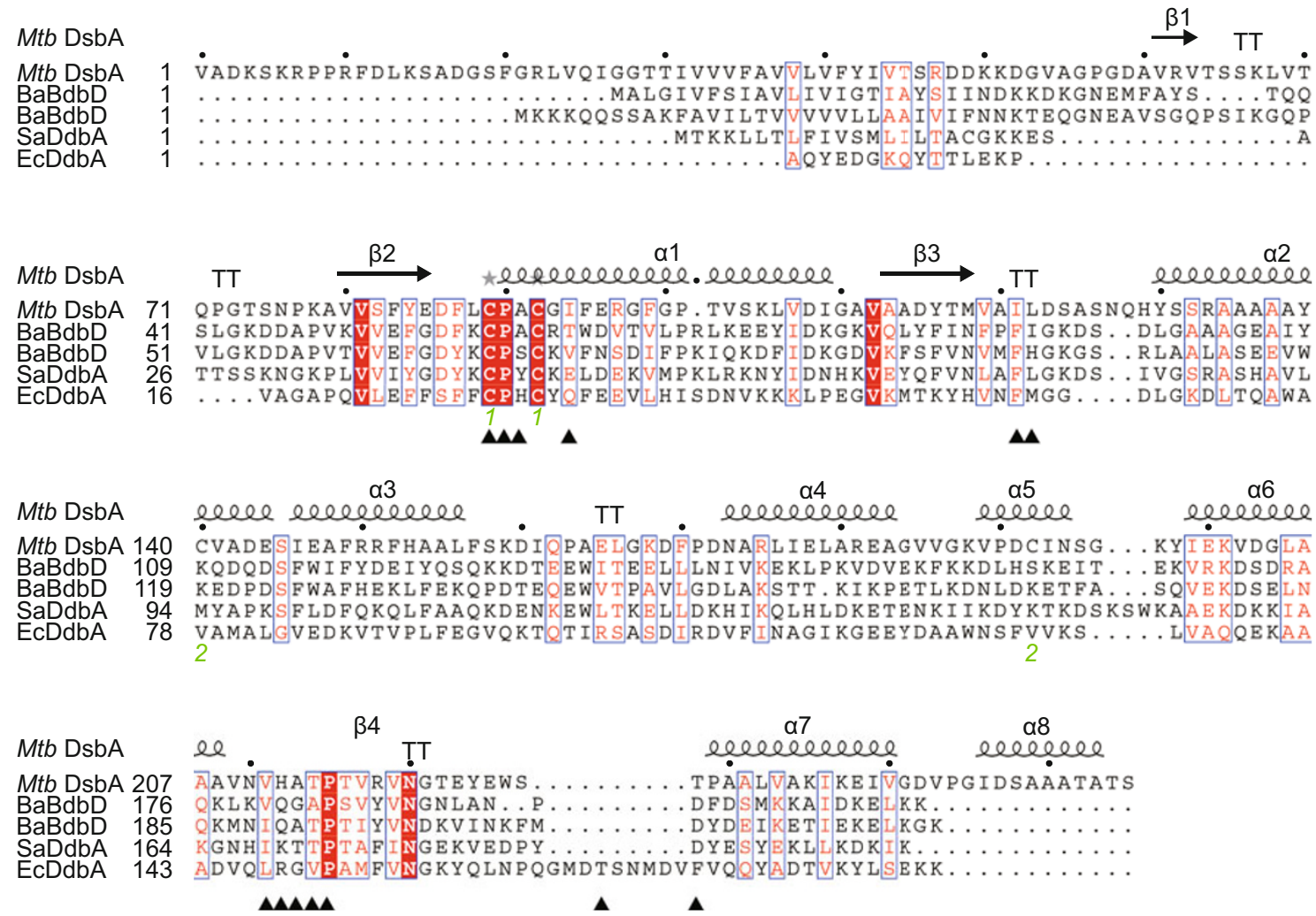

Figure 2. Multiple sequence alignment of DsbA homologues. Organisms: M.tuberculosis (Mtb), B.anthracis (Ba), B.subtilis (Bs), S.aureus (Sa, Gram-positive) and E.coli (Ec, Gram-negative). Secondary structures of Mtb DsbA are shown on the top and disulfide bonds were marked by numbers. The residues that form interactions at the EcDsbA-EcDsbB interface are labeled by triangle.

ent conformation of the loop3 in Mtb DsbA results in charged residues $\left(\mathrm{Glu}^{165}\right)$ covering this area, which flanks the active site. The different position of the loop4 in Mtb DsbA also results in an acidic protrusion (generated by $\mathrm{Glu}^{225}$ ) above the redox active site (left panel of Fig. 3D). The 9-residue deficiency and an extra a8 helix at the $C$ terminus of $M$ tb DsbA influence the surface properties in which the peptide-binding groove is truncated, and its position is changed when compared to EcDsbA. This groove was considered to be the interface of DsbA and $\mathrm{DsbB}$, and has been supposed to be involved in substrate binding (Inaba and Ito, 2008). We hypothesized that these variations in $M t b$ DsbA indicate different substrate specificity to that of EcDsbA.

\section{Active site dithiols}

The active site thioredoxin-like CXXC motif of Mtb DsbA is composed of $\mathrm{Cys}^{89}{ }^{8} \mathrm{Pro}^{90}-\mathrm{Ala}^{91}-\mathrm{Cys}^{92}$, and is located at the Nterminus of helix $\alpha 1$, where the two cysteines can interconvert between dithiol and disulfide forms. The active sites of the reduced and oxidized protein are shown in Fig. 4, together with an accompanying electron density map. The cysteines adopt a right-handed hook conformation, which is consistent with that of most active-site cysteines in the thioredoxin superfamily fold (Goulding et al., 2004). The conformation in which the cysteines are reduced has a distance of $3.35 \AA$ between the two Sy atoms (Fig. 4B). The Sy atom of $\mathrm{Cys}^{89}$ in the dithiol is exposed at the protein surface, and is stabilized by hydrogen bonds to both a water molecule $(3.53 \AA)$, and the side chain Oy atom of $\mathrm{Thr}^{214}$ (3.29 A). Meanwhile, Sy of Cys ${ }^{92}$ is buried and stabilized by weak hydrogen bonds to the $\mathrm{N}$ atom of cis$\operatorname{Pro}^{215}(3.68 \AA)$ and the side chain Oy atom of $\operatorname{Thr}^{214}(3.62 \AA)$. The cis-Pro loop is highly conserved among redox-active Trxfold superfamily members, and has been considered to be involved in substrate interactions (Kadokura et al., 2004). Mtb DsbA contains a TcP (Thr-cis Pro)-motif that is dominant in Gram-positive oxidases such as BdbD, but not in Gram-negative oxidases like EcDsbA (which generally possess a VcP motif). The conformation in which the cysteines are oxidized has a distance of $2.03 \AA$ between the two Sy atoms (Fig. 4C). The reduced form also appears to be present within the crystal structure of oxidized form, with occupancy of approximately $40 \%$, although Mtb DsbA was crystallized as a fully oxidized sample. Reduction of redox-active disulfides commonly occurs as a result of radiation damage (Weik et al., 2000; Alphey et al., 2003) and has been observed, e.g. in SaDsbA (Heras et al., 2008) and BdbD (Crow et al., 2009a). Comparing the forms of oxidized and reduced Mtb DsbA structure shows that there are small numbers of redox-linked conformational changes 


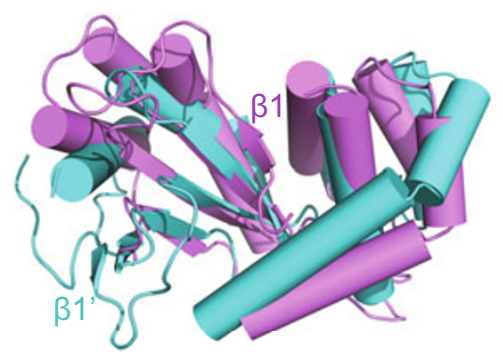

B

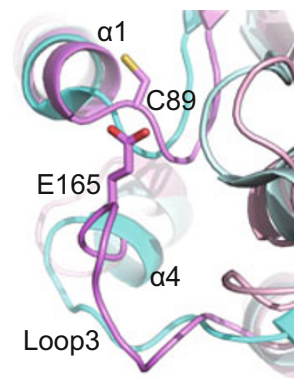

C

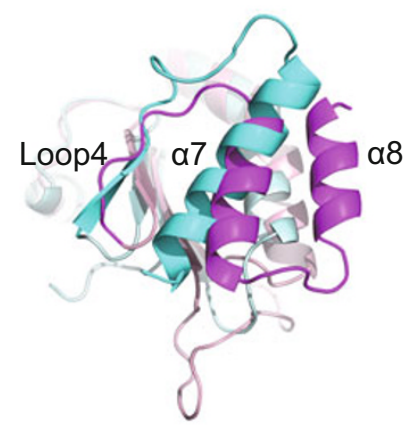

$\mathrm{E}$

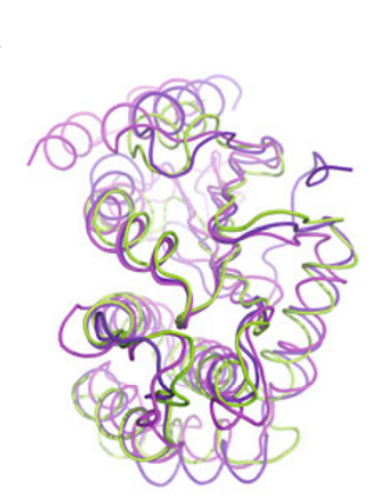

Mtb DsbA

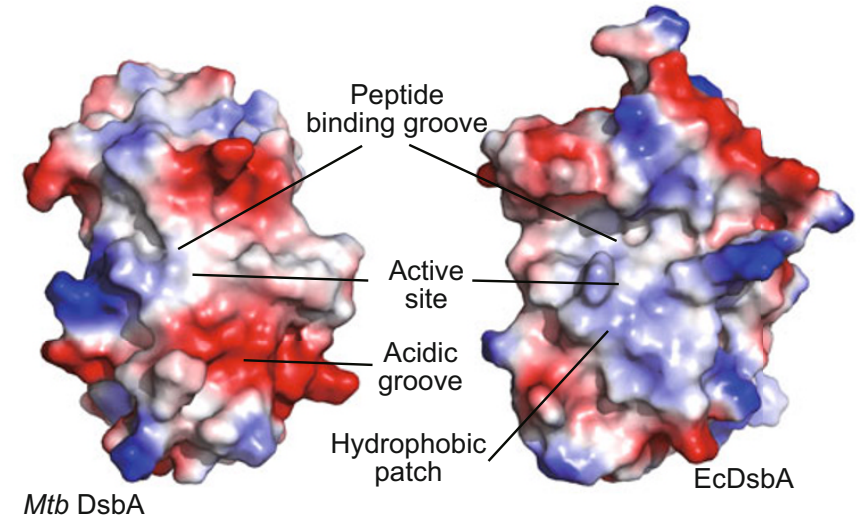

Figure 3. Comparison of $M t b$ DsbA with other DsbA proteins. (A) Superimposition of the structures of $M$ tb DsbA (magenta) and EcDsbA (cyan). $\beta 1$ is from Mtb DsbA, while $\beta 1$ ' is from EcDsbA. (B and C) Partial superimposition of Mtb DsbA (magenta) and EcDsbA (cyan). (D) Electrostatic surfaces of Mtb DsbA and EcDsbA are shown that exhibit the same orientation. Positive and negative electrostatic potentials are indicated in blue and red, respectively. (E) Superimposition of the structures of Mtb DsbA (magenta) and BsBdbD (purpleblue) and SaDsbA (green), which are shown as loop diagrams.

and the superposition of them gave an r.m.s.d. of $0.178 \AA$ (calculated for all atoms of residues 59-255).

\section{Oxidoreductase activity of Mtb DsbA}

In order to investigate the oxidative protein-folding activity of Mtb DsbA, we tested its ability to oxidize reduced and denatured RNase. The regeneration of native RNase from the reduced unfolded form to the fully oxidized native state was carried out in the presence and absence of Mtb DsbA, with the PDI (Protein disulfide isomerase) used as positive control. Native RNase can catalyze the hydrolysis of its cCMP substrate, which leads to change of absorbance at $296 \mathrm{~nm}$. In this assay, PDI catalyzed the refolding of RNase at a high rate, whereas refolding of RNase occurred at almost the same rate, regardless of Mtb DsbA being present or not (Fig. 5A).

To test Mtb DsbA's disulfide reductase activity, we performed a disulfide reductase activity assay with insulin as a substrate, which has two intramolecular disulfide bonds linking the $A$ and $B$ chains. When these disulfides are in their reduced state, the two chains will dissociate and the insoluble B chain will precipitate. Thus, we can test disulfide reductase activity by measuring the increase in turbidity of insulin solution. We measured the rate of insulin reduction by dithiothreitol (DTT) catalyzed by Mtb DsbA. Mtb DsbA did not show any activity, indicating that its surface features, which are less hydrophobic than those of EcDsbA, do not provide sufficient interaction with insulin (Fig. 5B).

\section{Models of the Mtb DsbA and Mtb VKOR complexes}

Mtb VKOR is a transmembrane protein with five transmembrane helices, with its $\mathrm{N}$-terminus localized in the cytoplasm, its C-terminus in the periplasm, and four cysteines facing towards the periplasm. Topology analysis indicates that the topology of Mtb VKOR is identical to that of Synechococcus sp. Thus, we used SpVKOR as model and FR-t5 method to construct the 3-D structure model of Mtb VKOR (Fig. 6B). Due to differences in the fifth helix of Mtb VKOR and SpVKOR, the model of the fifth helix in Mtb VKOR is not sufficiently accurate. According to the evaluation criteria of the FR-t5 method (Hu et al., 2011), the model is considered to be reasonable. Moreover, we choose eleven residues (51-61) (called the '1/2-segment') of the loop, located at the periplasmic side, between TM1 and TM2. This loop contains the cysteine whose corresponding residue in SpVKOR forms a disulfide bond with the cysteine of the CXXC motif of the Trx domain. Thus, we propose that the interaction between Mtb DsbA and Mtb VKOR occurs in the region sur- 
A

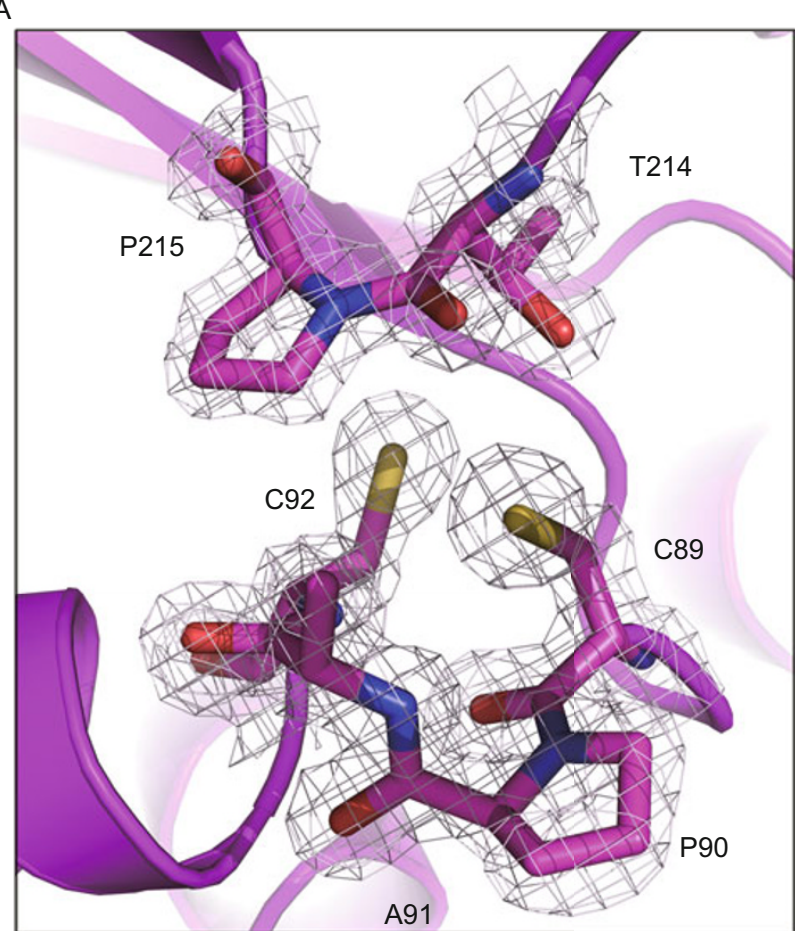

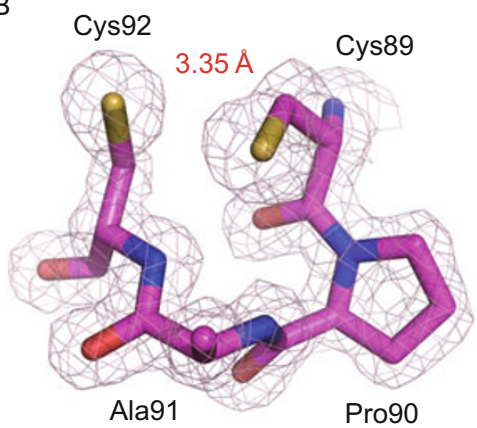

C

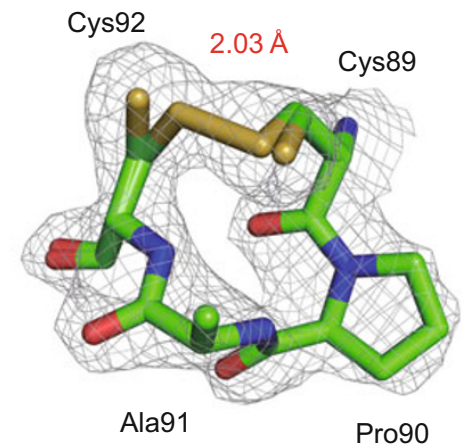

Figure 4. Active region of Mtb DsbA. (A) Detailed view of the N-terminus of helix a1 of Mtb DsbA showing the Cys-Pro-Ala-Cys active site of $M$ tb DsbA and the closely lying cis-proline $\left(\mathrm{Pro}^{215}\right)$ and Tyrosine $\left(\mathrm{Tyr}^{214}\right)$. (B and C) Electron density $(2 \mathrm{Fo}-\mathrm{Fc})$ of the active site region of $M t b$ DsbA in reduced and oxidized states, respectively (contoured at sigma 1.5).

A

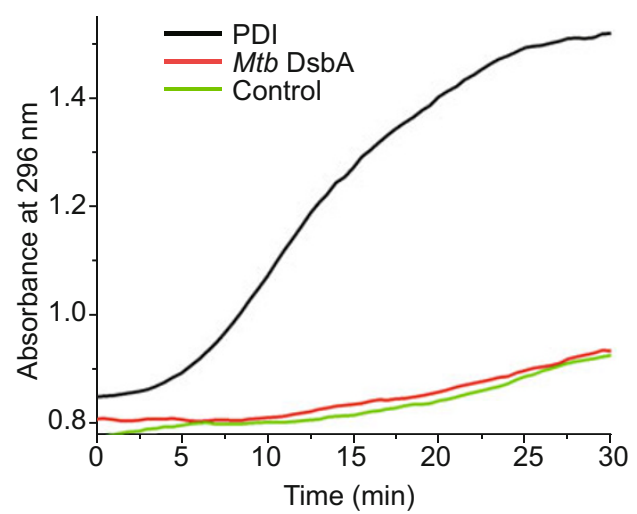

B

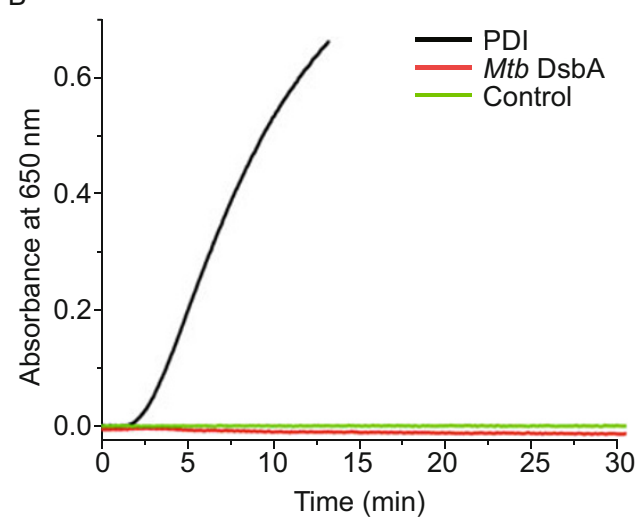

Figure 5. Oxidoreductase activity assay. (A) Reactivation of reduced and denatured RNase ( $8 \mu \mathrm{mol} / \mathrm{L})$ was incubated in $100 \mathrm{mmol} / \mathrm{L}$ Tris acetate buffer containing $50 \mathrm{mmol} / \mathrm{L} \mathrm{NaCl}, 1 \mathrm{mmol} / \mathrm{L} \mathrm{EDTA}, 1 \mathrm{mmol} / \mathrm{L} \mathrm{GSH}$, and $0.2 \mathrm{mmol} / \mathrm{L} \mathrm{GSSG}$ (pH 8.0), in the absence (green) or presence of a disulfide oxidoreductase $(0.5 \mu \mathrm{mol} / \mathrm{L}$ PDI (Black), $6 \mu \mathrm{mol} / \mathrm{L}$ Mtb DsbA (Red)). Cleavage of cCMP by native RNase A was analyzed spectroscopically at $296 \mathrm{~nm}$. (B) Insulin reduction assay. The reaction mixtures contained $130 \mu \mathrm{mol} / \mathrm{L}$ insulin in $0.1 \mathrm{~mol} / \mathrm{L}$ potassium phosphate buffer, $\mathrm{pH} 7.5,2.5 \mathrm{mmol} / \mathrm{L}$ EDTA. The reaction was performed in the absence (green) or presence of a disulfide oxidoreductase $(0.5 \mu \mathrm{mol} / \mathrm{L} \mathrm{PDI}$ (Black), $6 \mu \mathrm{mol} / \mathrm{L}$ Mtb DsbA (Red)). Reactions were initiated by adding DTT to a final concentration of $0.1 \mathrm{mmol} / \mathrm{L}$, and aggregation of reduced insulin was analyzed at $650 \mathrm{~nm}$ in a 6-s interval.

rounding the $\mathrm{Cys}^{57}$. Analysis of the autodock result between the 1/2-segment and Mtb DsbA, combined with the surface potential of the interface, we predicted a relatively credible and reasonable model of $M$ tb DsbA and Mtb VKOR (Fig. 6A). As shown in Fig. 6, the 1/2-segment of Mtb VKOR fitted into the active site groove of the Mtb DsbA Trx domain. In addition, previous structure alignment analyses demonstrated that this hydrophobic groove, which is formed by several loops between 
A

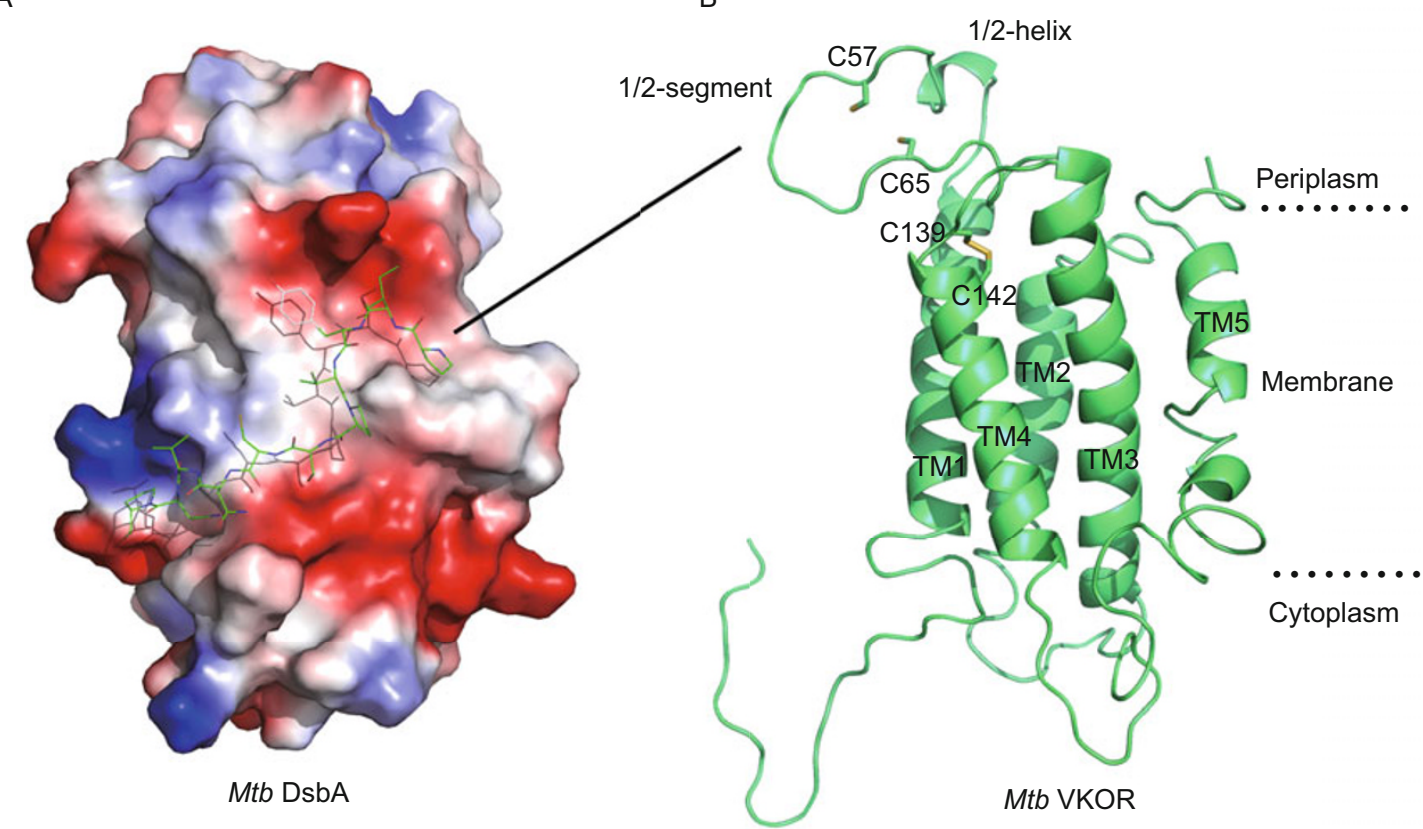

C

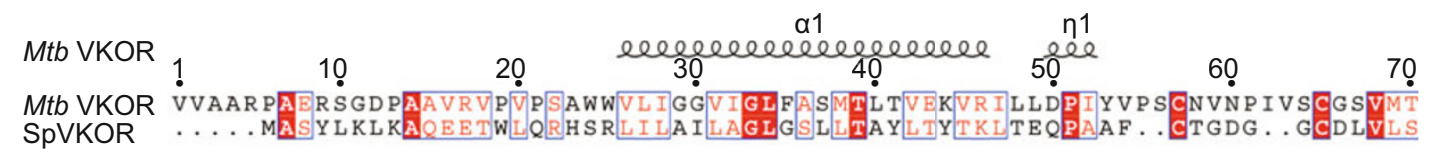

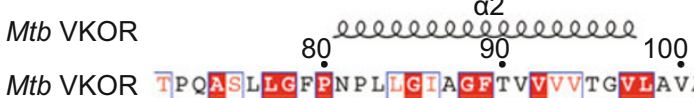

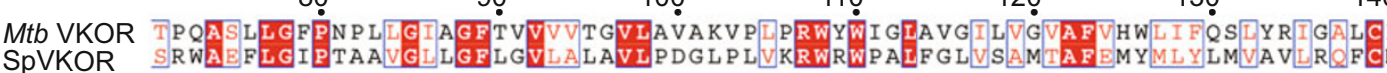

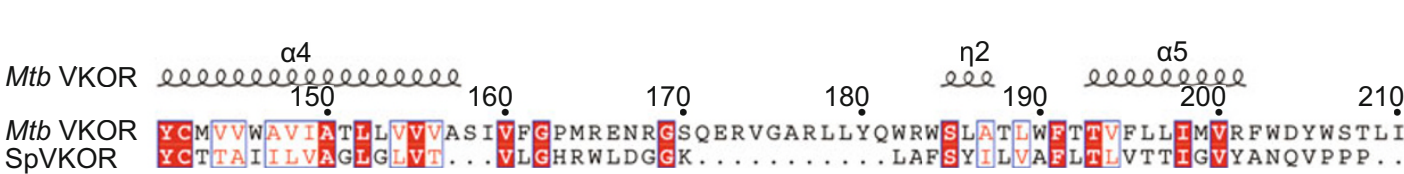

Figure 6. Complex model of interacting Mtb DsbA and Mtb VKOR. (A) Complex structure of Mtb DsbA and 1/2-segment of Mtb VKOR as modeled using Autodock Vina. Mtb DsbA is displayed as electrostatic surfaces, and 1/2-segment is shown as sticks representation (green). (B) The 3-D structure model of Mtb VKOR calculated using the FR-t5 threading method. (C) Sequence alignment of Mtb VKOR and SpVKOR. Secondary structures of Mtb VKOR are shown on the top.

the secondary structure elements and contains the active site CXXC motif, participates in the interaction between Mtb DsbA and VKOR.

\section{DISCUSSION}

Disulfide bonds, which are formed by the oxidation of two cysteine thiol groups, are important for the correct folding and stability of many secreted proteins (Depuydt et al., 2011). Cells of all organisms encode a series of proteins to participate in the formation of disulfide bonds. Bacterial Dsb proteins are known to promote the catalysis of disulfide bond formation. M.tuberculosis genome encodes several Dsb-like proteins besides DsbB orthologue. Until now, only two structures from M.tuberculosis had been determined: Mtb DsbE (a DsbA-like protein) and its homologue Mtb DsbF. Although Mtb DsbE is a $\mathrm{CcmG}$ (DsbE) homologue, which is a Gram-negative disulfide reductase, it has different functions. Mtb DsbE and DsbF can oxidatively refold reduced and unfolded hirudin, which is a small protein with three disulphide bonds in the native state (Hennecke et al., 1999; Goulding et al., 2004; Chim et al., 2010).

In the current study, we have determined two high-resolution crystal structures of $M t b D s b A$, one in its reduced, and one in its oxidized state. Like Mtb DsbE and DsbF, DsbA is also an extracellular protein with its $\mathrm{N}$-terminus anchoring in the mem- 
brane. Structural differences in the region between Mtb DsbA, $\mathrm{DsbE}$ and DsbF, which is surrounding the CXXC motif, indicate that they are likely to assist in correct folding of diverse sets of disulfide bonds of secreted or cell wall-associated proteins corresponding to diverse cellular conditions. Because of the absence of a periplasmic space, Gram-positive genomes encode a series of membrane-anchored Dsb proteins, which exhibit different structures and functions when compared to Dsb proteins expressed by Gram-negative bacteria. Thus, the catalytic mechanisms of Dsb proteins from E.coli should not be directly extended to these organisms.

Unlike EcDsbA, Mtb DsbA does not show any activity in the reduction of insulin or the oxidative refolding of RNase, which is typically used to characterize the redox activity of Thioredoxin fold proteins. Despite the overall structural similarity, Mtb DsbA does not appear to function like EcDsbA. The truncated binding groove, as well as an acidic rather than hydrophobic surface surrounding the CXXC motif, suggests that Mtb DsbA possesses different substrate specificity to EcDsbA. Furthermore, this region is also associated with the interaction between DsbA and its partner EcDsbB or Mtb VKOR. Structural analysis revealed that there are more residues between the two cysteines of loop1 in EcDsbB than present in VKOR. Thus, we speculate that EcDsbA and Mtb DsbA possess different surface features, to recognize and bind to their corresponding redox partners.

In the past 20 years, seventeen unique DsbA structures from different bacterial species have been solved (Shouldice et al., 2011). Amongst these structures, only Wolbachia pipientis a-DsbA1 possesses a second disulfide bond, which is highly conserved in a-proteobacterial DsbAs, not however, in other DsbAs. In Mtb DsbA, the second disulfide bond, which links a3 and $\alpha 5$ helices, is formed by Cys ${ }^{140}$ and Cys ${ }^{192}$. The oxidized forms of Mtb DsbA variant $(C 140,192 K)$ is less stable than its reduced form as well as its native protein. This was measured in two ways, a thermal stability assay (data not shown) and a pKa value determination assay. In the second assay, addition of hydrochloride to the oxidized Mtb DsbA variant solution resulted in instant protein precipitation, which resulted in difficulties in determining the $\mathrm{pKa}$ of $\mathrm{Cys}^{89}$. In addition, the disulfide bond in the active site of oxidized Mtb DsbA is a mixture of oxidized and reduced conformations. Reduction of redox-active disulfides is commonly caused by radiation damage. However, the second disulfide bond of Mtb DsbA remains oxidized in the crystal structure, suggesting that it is resistant to reducing conditions and is not redox-active, but contributes to the structural stability of the protein.

To date, the structures of two Gram-positive DsbA proteins have been solved, namely S.aureus DsbA, SaDsbA (PDB: $3 \mathrm{BCl}$ ) and B.subtilis BsDsbA (known as BdbD, PDB: 3EU3). Mtb DsbA shares some structural characteristics of SaDsbA and $\mathrm{BdbD}$ (Fig. 3E), especially the surface features surrounding the active site. Sequence alignment indicates that these features of Mtb DsbA are commonly conserved among Grampositive DsbAs. Thus, Gram-positive organisms may operate a common mechanism to form disulphide bonds. Moreover, an entire subgroup of Gram-positive bacteria lacks DsbB protein homologues; instead they contain VKOR, which is a functional alternative to DsbB.

VKOR plays an important role in the protein disulfide bondforming pathway in many bacteria (Dutton et al., 2010). The gene encoding DsbA in M.tuberculosis is directly adjacent to VKOR. According to the analysis of Mtb DsbA structure and the function of Mtb VKOR, we speculate that Mtb DsbA catalyzes the disulfide bond formation of substrate proteins, and then it is re-oxidized by Mtb VKOR, which in turn is re-oxidized by quinones present in the cytoplasm. Thus, we have attempted to express Mtb DsbA and VKOR in the E.coli expression system, both separately as well as together. However, we could not obtain satisfactory results from the overexpression studies of Mtb VKOR in E.coli, which may have been caused by differences in lipid composition of the cell membranes of E.coli and M.tuberculosis. Therefore, we used FR-t5 threading method in combination with Autodock Vina, to simulate the interaction between Mtb DsbA and VKOR. Based on membrane topology and mutational analysis of Mtb VKOR, we propose a disulphide formation pathway as presented in Fig. 7. We propose the following sequence of events: Cys ${ }^{89}$ and Cys ${ }^{92}$ of oxidized Mtb DsbA transfer their disulfide bond to an unfolded protein substrate; then $\mathrm{Cys}^{89}$ of reduced Mtb DsbA and Cys ${ }^{57}$ of $M$ tb VKOR can form a mixed disulfide bond, which is transferred onto Mtb DsbA; subsequently Cys ${ }^{65}$ of Mtb VKOR forms a disulfide bond with Cys ${ }^{139}$, resulting in re-oxidized $\mathrm{Cys}^{57}$ and Cys $^{65}$; and finally Cys ${ }^{139}$ and Cys ${ }^{142}$ are re-oxidized by a quinone in the cytoplasm.

Here, we have presented a structural study of $M t b$ DsbA, which suggests that it functions as a disulfide bond-forming protein. Biochemical analysis using an oxidoreductase activity assay indicates that it possesses different substrate specificities when compared to EcDsbA. The model of interaction between Mtb DsbA and VKOR will help in future investigations to elucidate the disulphide formation pathway of M.tuberculosis. Our study should provide an important first step in identifying the mechanisms for substrate recognition, and the details of electron transfer and disulfide bonds formation in M.tuberculosis. We believe that further structural and functional studies of Mtb DsbA and VKOR will contribute to the timely identification of much-needed, novel anti-TB drug targets.

\section{MATERIAL AND METHODS}

Protein expression and purification

The sequence encoding the M.tuberculosis Rv2969c' extracellular domain (residues 53-255 of Mtb DsbA, lacking the N-terminal single transmembrane helix) was amplified from M.tuberculosis $\mathrm{H}_{37} \mathrm{Rv}$ genomic DNA using two primers (forward, 5'-AGCCATATGGGCGTCGCGGGCCCCGGCGAT-3'; reverse, 5'-CCGCTCGAGTCAGGATGTCGCGGTAGCAGC-3'). The target sequence was inserted into the pET-28a protein expression vector (Novagen) at Ndel and $\mathrm{Xhol}$ restriction sites (underscored in the primers). Escherichia 


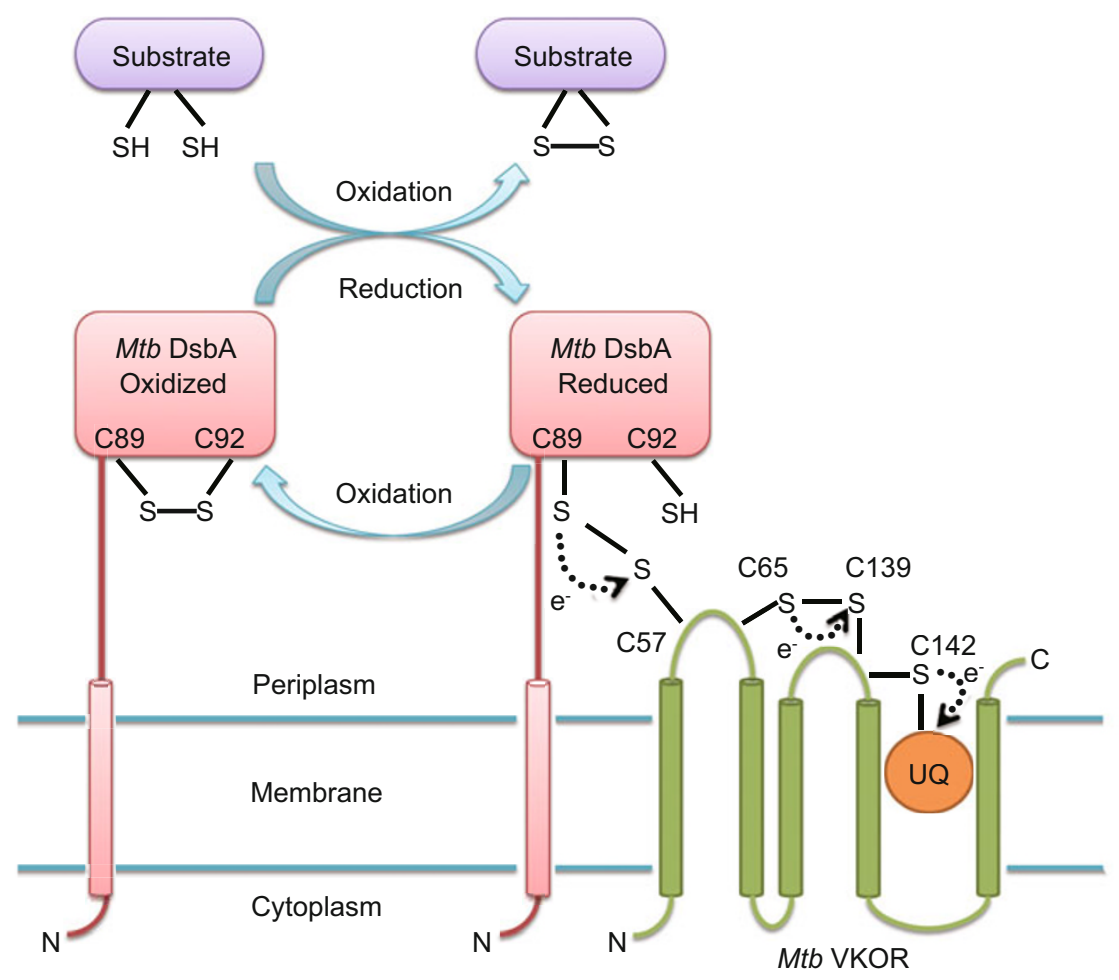

Figure 7. Disulphide formation pathway in M.tuberculosis.

coli C43 (DE3) cells were transformed with the recombinant vector for expression. For Se-derivative protein expression, a mutant form of Mtb DsbA (Lys156, 232Met) was generated by site-directed mutagenesis to improve SeMet incorporation and anomalous signal for SeMet derivative protein. For biochemical properties assays, another mutant form of Mtb DsbA (Cys140, 192Lys) was constructed and expressed. Recombinant E.coli cells were cultured aerobically at $37^{\circ} \mathrm{C}$ for $4-5 \mathrm{~h}$ in Terrific Broth medium containing $50 \mu \mathrm{g} / \mathrm{mL}$ kanamycin. When the OD600 reached $\sim 0.8$, cells were cultured at $16^{\circ} \mathrm{C}$ for $16-20 \mathrm{~h}$ after induction with $0.5 \mathrm{mmol} / \mathrm{L}$ Isopropyl $\beta$-D-1-thiogalactopyranoside. Cells were harvested at $4,670 \mathrm{~g}$ for $30 \mathrm{~min}$ at $4^{\circ} \mathrm{C}$; resuspended in $20 \mathrm{mmol} / \mathrm{L} \mathrm{Tris}-\mathrm{HCl}$ (pH 8.0), $150 \mathrm{mmol} / \mathrm{L} \mathrm{NaCl}, 10 \%$ glycerol $(\mathrm{v} / \mathrm{V})$. Cells were disrupted by High voltage breaker (AVESTIN Company, EF-C3) and the lysate was centrifuged at $30,700 \mathrm{~g}$ for $30 \mathrm{~min}$ at $4^{\circ} \mathrm{C}$ to remove unbroken cells and debris.

The supernatant was applied twice to a nickel affinity column (Qiagen) equilibrated in $20 \mathrm{mmol} / \mathrm{L}$ Tris- $\mathrm{HCl}(\mathrm{pH} 8.0), 150 \mathrm{mmol} / \mathrm{L} \mathrm{NaCl}$, $10 \%$ glycerol, $20 \mathrm{mmol} / \mathrm{L}$ imidazole, and washed with $20 \mathrm{mmol} / \mathrm{L}$ Tris$\mathrm{HCl}(\mathrm{pH} 8.0), 150 \mathrm{mmol} / \mathrm{L} \mathrm{NaCl}, 10 \%$ glycerol, $50 \mathrm{mmol} / \mathrm{L}$ imidazole to remove proteins bound nonspecifically. The recombinant $M t b$ DsbA was eluted with $20 \mathrm{mmol} / \mathrm{L}$ Tris- $\mathrm{HCl}(\mathrm{pH} 8.0), 150 \mathrm{mmol} / \mathrm{L} \mathrm{NaCl}, 10 \%$ glycerol, $200 \mathrm{mmol} / \mathrm{L}$ imidazole. The target protein was further purified using a Hitrap $Q$ anion exchange column (GE Healthcare) and a Superdex-75 gel filtration column (GE Healthcare). Purified Mtb DsbA (native or Se-derived) was concentrated to $7.5 \mathrm{mg} / \mathrm{mL}$ in $20 \mathrm{mmol} / \mathrm{L}$ Tris- $\mathrm{HCl}$ ( $\mathrm{pH} 8.0), 150 \mathrm{mmol} / \mathrm{L} \mathrm{NaCl}$ for crystallization trails.

\section{Oxidation and reduction of Mtb DsbA}

To oxidize and reduce Mtb DsbA, 1.7 mmol/L Dichloro (1,10-phenan- throline) copper (II) and $100 \mathrm{mmol} / \mathrm{L}$ dithiothreitol (DTT) were added to $M$ tb DsbA (approximately $20 \mathrm{mmol} / \mathrm{L}$ ), respectively, in $150 \mathrm{mmol} / \mathrm{L}$ $\mathrm{NaCl}$ and $20 \mathrm{mmol} / \mathrm{L}$ Tris- $\mathrm{HCl}, \mathrm{pH} 8.0$, and incubated overnight at $4^{\circ} \mathrm{C}$. The oxidized and reduced proteins were then isolated using a HiTrap ${ }^{\circledR}$ Desalting column (GE Healthcare) equilibrated in their original buffer. Under non-reducing conditions, oxidized proteins migrate faster on an SDS-PAGE gel than reduced proteins. Therefore, samples of the treated $M t b$ DsbA were run on an SDS-PAGE gel with no DTT present, to ensure that $M t b$ DsbA was either fully oxidized or fully reduced.

\section{Crystallization}

Crystallization of the Mtb DsbA was performed at $16^{\circ} \mathrm{C}$ by the hanging drop vapor diffusion method. Equal volumes $(1.5 \mu \mathrm{L})$ of protein and crystallization reagent (30\%-35\% (w/v) PEG 2000, 0.2 mol/L Ammonium Sulfate, $0.1 \mathrm{~mol} / \mathrm{L}$ Sodium Acetate trihydrate, $\mathrm{pH}$ 4.3-4.6) were mixed and equilibrated over a $200 \mu \mathrm{L}$ reservoir solution. Twined, platelike crystals were obtained with poor diffraction quality. Using initial crystals as seeds, single plate-like crystals grew in the drops containing lower concentrations of $26 \%-28 \%$ (w/V) PEG 2000. The crystal used for data collection was grown in 28\% (w/v) PEG 2000, $0.2 \mathrm{~mol} / \mathrm{L}$ Ammonium Sulfate, $0.1 \mathrm{~mol} / \mathrm{L}$ Sodium Acetate trihydrate, $\mathrm{pH}$ 4.3. The Se-Mtb DsbA derivative and oxidized crystals were obtained using the reduced protein crystals as seeds under identical conditions.

\section{Data collection and processing}

A set of single-wavelength anomalous dispersion data for Se-Met crystals was collected at BL5A of Photon Factory (Japan) at a wavelength of $0.9790 \AA$. About $15 \%(\mathrm{~V} / \mathrm{V})$ glycerol was added to the reservoir solution as a cryoprotectant for the crystals measured. Native diffraction 
data for the oxidized Mtb DsbA crystals were collected at BL17A of Photon Factory (Japan) at a wavelength of $0.9800 \AA$. Data processing for both native and Se-Met crystals was carried out using the HKL2000 program package (Otwinowski and Minor, 1997).

\section{Structure determination and refinement}

We used the Phenix program package (Emsley and Cowtan, 2004) to solve the phases of the reduced Mtb DsbA protein utilizing the SAD data, and built $\sim 90 \%$ of the structure of the reduced $M t b$ DsbA protein with the automated program. The missing structure was manually added and adjusted in COOT (Emsley et al., 2010). Phenix was used for structure refinement. Water molecules were then added in cases where there were $>3 \sigma$ (Fo-Fc) electron density, and where good hydrogen bonds with nearby residues could be formed. The final structure of reduced Mtb DsbA was validated with PROCHECK, indicating that $99 \%$ of all residues lie in the favored regions of the Ramachandran Plot, with $1.0 \%$ of residues located in allowed regions, and no residues in disallowed regions. The oxidized state structure of Mtb DsbA was solved by the Molecular Replacement method, using the reduced Mtb $D s b A$ structure as model. Protein structures were viewed, and pictures were made using the PyMOL software (DeLano, 2002).

\section{In vitro oxidoreductase activity assays}

The preparation of reduced, denatured RNase, and the subsequent refolding assays were performed as described previously by Wang et al. (2010). Briefly, $8 \mu \mathrm{mol} / \mathrm{L}$ reduced and denatured RNase was incubated with $6 \mu \mathrm{mol} / \mathrm{L}$ oxidized Mtb DsbA in glutathione redox buffer (100 mmol/L Tris acetate buffer containing $50 \mathrm{mmol} / \mathrm{L} \mathrm{NaCl}, 1 \mathrm{mmol} / \mathrm{L}$ EDTA, $1 \mathrm{mmol} / \mathrm{L} \mathrm{GSH}$ (glutathione), and $0.2 \mathrm{mmol} / \mathrm{L} \mathrm{GSSG}$ (glutathione disulfide) $\mathrm{pH}$ 8.0). The assay was performed at $25^{\circ} \mathrm{C}$, and samples were recorded every 6 seconds; RNase activity was determined by monitoring the absorbance increase at $296 \mathrm{~nm}$ due to cCMP hydrolysis. Insulin was added to a final concentration of $130 \mu \mathrm{mol} / \mathrm{L}$ to $0.1 \mathrm{~mol} / \mathrm{L}$ potassium phosphate buffer containing $2.5 \mathrm{mmol} / \mathrm{L}$ EDTA, $0.1 \mathrm{mmol} / \mathrm{L}$ DTT $(\mathrm{pH} 7.5)$ in the presence of $6 \mu \mathrm{mol} / \mathrm{L}$ reduced $M t b$ $\mathrm{DsbA}$ to initiate the reaction at $25^{\circ} \mathrm{C}$. The measurements for the reaction mixture were performed in a spectrophotometer at $650 \mathrm{~nm}$, detecting light scattering from reduced and precipitated insulin chains, and data were collected every $6 \mathrm{~s}$. One reaction using treated RNase in the presence of PDI was used as positive control (Wang et al., 2010), while treated RNase in the absence of any oxidoreductase served as negative control reaction.

\section{Construction of the protein complex structure}

Modeling of protein structures was carried out using experimentally determined structures as structure templates, a process called template based modeling. To this end, we used a known crystal structure of VKOR from Synechococcus $s p$., which exhibits local sequence identity with Mtb VKOR of about 30\% (28/94), in combination with a threading method called FR-t5 (fold recognition by use of 5 terms) (Hu et al., 2011) to construct the model of Mtb VKOR. Analysis of the structures of SpVKOR and EcDabA-DabB complex (Inaba et al., 2006) indicates the features and the residues that form interactions at the protein-protein interface. Therefore we chose eleven residues (51-61) from the loop between $\alpha 1$ and $\alpha 2$ of Mtb VKOR, and used Autodock Vina (Trott and Olson, 2010) to construct the model of the interaction between Mtb DsbA and VKOR.

\section{DEPOSITION OF COORDINATES}

Coordinates and structure factors for the crystal structure of the Mtb DsbA have been deposited to the Research Collaboratory for Structural Bioinformatics Protein Data Bank (http://www.rcsb.org/pdb/). The entries are 4JR4 for the oxidized form and 4JR6 for the reduced form.

\section{ACKNOWLEDGEMENTS}

The authors thank Ya Wang, Yi Han, Xiaoxia Yu, and other staff members of the Structural Biology Core Facility in the Institute of Biophysics, Chinese Academy of Sciences (CAS) for their excellent technical work. This work was supported by grants from the State Key Development Program for Basic Research of the Ministry of Science and Technology of China (973 Project) (Grant Nos. 2011CB915501 and 2011CB910304), and the National Infectious Disease Funding (Grant No. 2012ZX10004701).

\section{ABBREVIATIONS}

Dsb, disulfide bond-forming proteins; DTT, dithiothreitol; E. coli, Escherichia coli; FR-t5, fold recognition by use of 5 terms; Mtb, Mycobacterium tuberculosis; PDI, Protein disulfide isomerase; r.m.s.d., root mean square deviation function; $\mathrm{SAD}$, single anomalous diffraction; $\mathrm{TB}$, tuberculosis; VKOR, Vitamin K epoxide reductase

\section{COMPLIANCE WITH ETHICS GUIDELINES}

Lu Wang, Jun Li, Xiangxi Wang, Wu Liu, Xuejun C.zhang, Xuemei Li, Zihe Rao declare that they have no conflict of interest.

This article does not contain any studies with human or animal subjects performed by the any of the authors.

\section{REFERENCES}

Alphey, M.S., Gabrielsen, M., Micossi, E., Leonard, G.A., McSweeney, S.M., Ravelli, R.B., Tetaud, E., Fairlamb, A.H., Bond, C.S., and Hunter, W.N. (2003). Tryparedoxins from Crithidia fasciculata and Trypanosoma brucei: photoreduction of the redox disulfide using synchrotron radiation and evidence for a conformational switch implicated in function. J Biol Chem 278, 25919-25925.

Chim, N., Riley, R., The, J., Im, S., Segelke, B., Lekin, T., Yu, M., Hung, L.W., Terwilliger, T., Whitelegge, J.P., et al. (2010). An extracellular disulfide bond forming protein (DsbF) from Mycobacterium tuberculosis: structural, biochemical, and gene expression analysis. J Mol Biol 396, 1211-1226.

Comas, I., and Gagneux, S. (2009). The past and future of tuberculosis research. PLoS Pathog 5, e1000600.

Crow, A., Lewin, A., Hecht, O., Carlsson Moller, M., Moore, G.R., Hederstedt, L., and Le Brun, N.E. (2009a). Crystal structure and biophysical properties of Bacillus subtilis $\mathrm{BdbD}$. An oxidizing thiol:disulfide oxidoreductase containing a novel metal site. J Biol Chem 284, 23719-23733.

Crow, A., Liu, Y., Moller, M.C., Le Brun, N.E., and Hederstedt, L. (2009b). Structure and functional properties of Bacillus subtilis en- 
dospore biogenesis factor StoA. J Biol Chem 284, 10056-10066.

Daniels, R., Mellroth, P., Bernsel, A., Neiers, F., Normark, S., von Heijne, G., and Henriques-Normark, B. (2010). Disulfide bond formation and cysteine exclusion in gram-positive bacteria. $\mathrm{J}$ Biol Chem 285, 3300-3309.

DeLano, W.L. (2002). The PyMOL Molecular Graphics System. DeLano Scientific, San Carlos, CA, USA.

Denoncin, K., and Collet, J.F. (2012). Disulfide bond formation in the bacterial periplasm: major achievements and challenges ahead. Antioxid Redox Signal 19, 63-71.

Depuydt, M., Messens, J., and Collet, J.F. (2011). How proteins form disulfide bonds. Antioxid Redox Signal 15, 49-66.

Dorenbos, R., Stein, T., Kabel, J., Bruand, C., Bolhuis, A., Bron, S., Quax, W.J., and Van Dijl, J.M. (2002). Thiol-disulfide oxidoreductases are essential for the production of the lantibiotic sublancin 168. J Biol Chem 277, 16682-16688.

Dumoulin, A., Grauschopf, U., Bischoff, M., Thony-Meyer, L., and Berger-Bachi, B. (2005). Staphylococcus aureus DsbA is a membranebound lipoprotein with thiol-disulfide oxidoreductase activity. Arch Microbiol 184, 117-128.

Dutton, R.J., Boyd, D., Berkmen, M., and Beckwith, J. (2008). Bacterial species exhibit diversity in their mechanisms and capacity for protein disulfide bond formation. Proc Natl Acad Sci U S A 105, 11933-11938.

Dutton, R.J., Wayman, A., Wei, J.R., Rubin, E.J., Beckwith, J., and Boyd, D. (2010). Inhibition of bacterial disulfide bond formation by the anticoagulant warfarin. Proc Natl Acad Sci U S A 107, 297-301.

Emsley, P., and Cowtan, K. (2004). Coot: model-building tools for molecular graphics. Acta Crystallogr D Biol Crystallogr 60, 2126-2132.

Emsley, P., Lohkamp, B., Scott, W.G., and Cowtan, K. (2010). Features and development of Coot. Acta Crystallogr D Biol Crystallogr 66, 486-501.

Ericsson, U.B., Hallberg, B.M., DeTitta, G.T., Dekker, N., and Nordlund, P. (2006). Thermofluor-based high-throughput stability optimization of proteins for structural studies. Anal Biochem 357, 289-298.

Erlendsson, L.S., Acheson, R.M., Hederstedt, L., and Le Brun, N.E. (2003). Bacillus subtilis ResA is a thiol-disulfide oxidoreductase involved in cytochrome c synthesis. J Biol Chem 278, 17852-17858.

Erlendsson, L.S., and Hederstedt, L. (2002). Mutations in the thiol-disulfide oxidoreductases $\mathrm{BdbC}$ and $\mathrm{BdbD}$ can suppress cytochrome c deficiency of CcdA-defective Bacillus subtilis cells. J Bacteriol 184, 1423-1429.

Goldstone, D., Baker, E.N., and Metcalf, P. (2005). Crystallization and preliminary diffraction studies of the C-terminal domain of the DipZ homologue from Mycobacterium tuberculosis. Acta Crystallogr Sect F Struct Biol Cryst Commun 61, 243-245.

Goulding, C.W., Apostol, M., Anderson, D.H., Gill, H.S., Smith, C.V., Kuo, M.R., Yang, J.K., Waldo, G.S., Suh, S.W., Chauhan, R., et al. (2002). The TB structural genomics consortium: providing a structural foundation for drug discovery. Curr Drug Targets Infect Disord 2, 121-141.

Goulding, C.W., Apostol, M.I., Gleiter, S., Parseghian, A., Bardwell, J., Gennaro, M., and Eisenberg, D. (2004). Gram-positive DsbE proteins function differently from Gram-negative DsbE homologs. A structure to function analysis of DsbE from Mycobacterium tuberculosis. J Biol Chem 279, 3516-3524.

Goulding, C.W., Perry, L.J., Anderson, D., Sawaya, M.R., Cascio, D.,
Apostol, M.I., Chan, S., Parseghian, A., Wang, S.S., Wu, Y., et al. (2003). Structural genomics of Mycobacterium tuberculosis: a preliminary report of progress at UCLA. Biophys Chem 105, 361-370.

Hennecke, J., Sebbel, P., and Glockshuber, R. (1999). Random circular permutation of DsbA reveals segments that are essential for protein folding and stability. J Mol Biol 286, 1197-1215.

Heras, B., Kurz, M., Jarrott, R., Shouldice, S.R., Frei, P., Robin, G., Cemazar, M., Thony-Meyer, L., Glockshuber, R., and Martin, J.L. (2008). Staphylococcus aureus DsbA does not have a destabilizing disulfide. A new paradigm for bacterial oxidative folding. J Biol Chem 283, 4261-4271.

Hu, Y., Dong, X., Wu, A., Cao, Y., Tian, L., and Jiang, T. (2011). Incorporation of local structural preference potential improves fold recognition. PLoS One 6, e17215.

Inaba, K., and Ito, K. (2008). Structure and mechanisms of the DsbBDsbA disulfide bond generation machine. Biochim Biophys Acta 1783, 520-529.

Inaba, K., Murakami, S., Suzuki, M., Nakagawa, A., Yamashita, E., Okada, K., and Ito, K. (2006). Crystal structure of the DsbB-DsbA complex reveals a mechanism of disulfide bond generation. Cell 127, 789-801.

Kadokura, H., Tian, H., Zander, T., Bardwell, J.C., and Beckwith, J. (2004). Snapshots of DsbA in action: detection of proteins in the process of oxidative folding. Science 303, 534-537.

Krissinel, E., and Henrick, K. (2007). Inference of macromolecular assemblies from crystalline state. J Mol Biol 372, 774-797.

Kurz, M., Iturbe-Ormaetxe, I., Jarrott, R., Shouldice, S.R., Wouters, M.A., Frei, P., Glockshuber, R., O'Neill, S.L., Heras, B., and Martin, J.L. (2009). Structural and functional characterization of the oxidoreductase alpha-DsbA1 from Wolbachia pipientis. Antioxid Redox Signal 11, 1485-1500.

Li, W., Schulman, S., Dutton, R.J., Boyd, D., Beckwith, J., and Rapoport, T.A. (2010). Structure of a bacterial homologue of vitamin K epoxide reductase. Nature 463, 507-512.

Martin, J.L., Bardwell, J.C., and Kuriyan, J. (1993). Crystal structure of the DsbA protein required for disulphide bond formation in vivo. Nature 365, 464-468.

Matthews, B.W. (1968). Solvent content of protein crystals. J Mol Biol 33, 491-497.

McCarthy, A.A., Haebel, P.W., Torronen, A., Rybin, V., Baker, E.N., and Metcalf, P. (2000). Crystal structure of the protein disulfide bond isomerase, DsbC, from Escherichia coli. Nat Struct Biol 7, 196-199.

Meima, R., Eschevins, C., Fillinger, S., Bolhuis, A., Hamoen, L.W., Dorenbos, R., Quax, W.J., van Dijl, J.M., Provvedi, R., Chen, I., et al. (2002). The bdbDC operon of Bacillus subtilis encodes thioldisulfide oxidoreductases required for competence development. J Biol Chem 277, 6994-7001.

Messens, J., and Collet, J.F. (2006). Pathways of disulfide bond formation in Escherichia coli. Int J Biochem Cell Biol 38, 1050-1062.

Otwinowski, Z., and Minor, W. (1997). Processing of X-ray diffraction data collected in oscillation mode. Method Enzymol 276, 307-326.

Paxman, J.J., Borg, N.A., Horne, J., Thompson, P.E., Chin, Y., Sharma, P., Simpson, J.S., Wielens, J., Piek, S., Kahler, C.M., et al. (2009). The structure of the bacterial oxidoreductase enzyme DsbA in complex with a peptide reveals a basis for substrate specificity in the catalytic cycle of DsbA enzymes. J Biol Chem 284, 17835-17845.

Reid, E., Cole, J., and Eaves, D.J. (2001). The Escherichia coli CcmG 
protein fulfils a specific role in cytochrome $\mathrm{c}$ assembly. Biochem $\mathrm{J}$ 355, 51-58.

Shao, F., Bader, M.W., Jakob, U., and Bardwell, J.C. (2000). DsbG, a protein disulfide isomerase with chaperone activity. J Biol Chem 275, 13349-13352.

Shouldice, S.R., Heras, B., Walden, P.M., Totsika, M., Schembri, M.A., and Martin, J.L. (2011). Structure and function of DsbA, a key bacterial oxidative folding catalyst. Antioxid Redox Signal 14, 1729-1760.

Stewart, E.J., Katzen, F., and Beckwith, J. (1999). Six conserved cysteines of the membrane protein $\mathrm{DsbD}$ are required for the transfer of electrons from the cytoplasm to the periplasm of Escherichia coli. EMBO J 18, 5963-5971.

Trott, O., and Olson, A.J. (2010). AutoDock Vina: improving the speed and accuracy of docking with a new scoring function, efficient optimization, and multithreading. J Comput Chem 31, 455-461.
Wang, C., Chen, S., Wang, X., Wang, L., Wallis, A.K., Freedman, R.B., and Wang, C.C. (2010). Plasticity of human protein disulfide isomerase: evidence for mobility around the $\mathrm{X}$-linker region and its functional significance. J Biol Chem 285, 26788-26797.

Wang, X., Dutton, R.J., Beckwith, J., and Boyd, D. (2011). Membrane topology and mutational analysis of Mycobacterium tuberculosis VKOR, a protein involved in disulfide bond formation and a homologue of human vitamin $\mathrm{K}$ epoxide reductase. Antioxid Redox Signal 14, 1413-1420.

Weik, M., Ravelli, R.B., Kryger, G., McSweeney, S., Raves, M.L., Harel, M., Gros, P., Silman, I., Kroon, J., and Sussman, J.L. (2000). Specific chemical and structural damage to proteins produced by synchrotron radiation. Proc Natl Acad Sci U S A 97, 623-628.

Yang, Q., Yu, K., Yan, L., Li, Y., Chen, C., and Li, X. (2011). Structural view of the regulatory subunit of aspartate kinase from Mycobacterium tuberculosis. Protein Cell 2, 745-754. 